\title{
Surface Science and Some of the Surface Analytical Techniques
}

\author{
Ananta Raj Acharya \\ Email: acharyaannata@yahoo.com
}

Surface science is the study of physical and chemical phenomena that occur at the interface. The term surface science came into common parlance in the early 1960s. Although there had been many previous studies of the surfaces and surface phenomena, some of which led to the Nobel prize winning work of Langmuir for his studies of adsorption and of Davison and Germer on the demonstration of low energy electron diffraction(LEED). These studies were generally classified under various other scientific sub disciplines, such as physical chemistry or electron physics.

Since its inception, field of surface science has undergone an explosive expansion. This expansion has been driven by the combination of the ready availability of ultra high vacuum environments, the development of techniques for the preparation of macroscopic single crystal surfaces and the application of an increasingly complex array of surface analytical techniques, which have made possible characterization of the structure and reactivity of a wide range of surfaces. For most surface science experiments, Ultra High Vacuum (UHV) is required for the two principal reasons: (1) to enable atomically clean surfaces to be prepared for study and such surfaces to be maintained in a contamination-free state for the duration of the experiment and (2) to permit the use of low energy electron and ion-based experimental techniques without undue interference from the gas phase scattering. Early work was carried out in glass vacuum systems using liquid nitrogen trapped $\mathrm{Hg}$ diffusion pumps. The surface science instrumentation had to be incorporated into these sealed glass vessels with electrical connections made through glass-to-metal seals in the containment vessel. Modern surface science studies usually involve the use of many different techniques in the same level, each of which may be quite sophisticated and this is achieved by mounting each onto a stainless steel flange which is sealed to a stainless steel chamber using $\mathrm{Cu}$ gasket seals. In addition to the development of these metal vessels, great use is now made of ion pumps which

require only electrical power to function and do not need liquid nitrogen. Although one must use vacuum pumps capable of operating in the $10^{-10}-10^{-11}$ torr range, an important ingredient in obtaining UHV is the need to 'bake' the whole system. Typically, a stainless steel chamber with all its enclosed instrumentation is baked to $200{ }^{\circ} \mathrm{C}$ for 12 hours or so. Obviously this means that all components in the vacuum chamber must be stable and have low vapor pressures at $200{ }^{\circ} \mathrm{C}$. An additional requirement for the experiments is that all components must be non-magnetic as many surface techniques involve low energy electrons which are easily deflected by weak electrostatic and magnetic fields. Fabrication methods compatible with these requirements are now well established involving mainly the use of stainless steel and refractory metals with ceramics for electrical or thermal insulation. Many materials acceptable in 'high vacuum' ( $\left.10^{-6} \mathrm{Torr}\right)$ such as many adhesives and plastics are not acceptable in UHV.

While UHV guarantees that a surface should not be influenced by the arrival of ambient atoms and molecules on a time scale of the order of one hour or more, a further requirement to studies of the properties of ideal surfaces is to be able to clean them in the vacuum system. The main methods used to achieve this in situ cleaning are: heating, ion bombardment (typically Ar ions) and chemical processing.

Heating a surface, like heating the walls of a vacuum chamber, can lead to desorption of adsorbed species. However, in most cases some impurities on the surface are too strongly bound to be removed by heating to the temperatures below the melting point of the sample. The use of Ar ion bombardment of a surface to remove layers of the surface by sputtering is by far the most widely used primary method, 
particularly for metal surfaces. This technique is effective in the removal of many atomic layers of a surface. One disadvantage of ion bombardment, typically at energies of $0.5-5 \mathrm{keV}$, is that the surface is left in a heavily damaged state, usually with embedded Ar atoms, so that the surface must be annealed to restore the order. The final approach of chemical cleaning in situ involves the introduction of gases into the vacuum system at low pressures which react with impurities on a surface to produce weakly bound species which can be thermally desorbed.

Once the sample is cleaned, the surface characterization of the sample can be done using different techniques. A few techniques are described below.

\section{Auger Electron Spectroscopy}

Pierre Victor Auger, a French scientist, discovered the Auger effect in 1925 upon analysis of a Wilson cloud chamber experiment. The term 'Auger effect' comes from his name. When an incident electron of sufficient energy knocks an electron out from the core level of an atom, a vacancy is created. This vacancy is fulfilled by the jump of another electron from the higher level releasing a quantum of energy. Although sometimes this energy is released in the form of an emitted photon, the energy can also be transferred to another electron, which is ejected from the atom. This second ejected electron is called an Auger electron. The process of emission of Auger electron can be visualized with the help of the following diagrams.
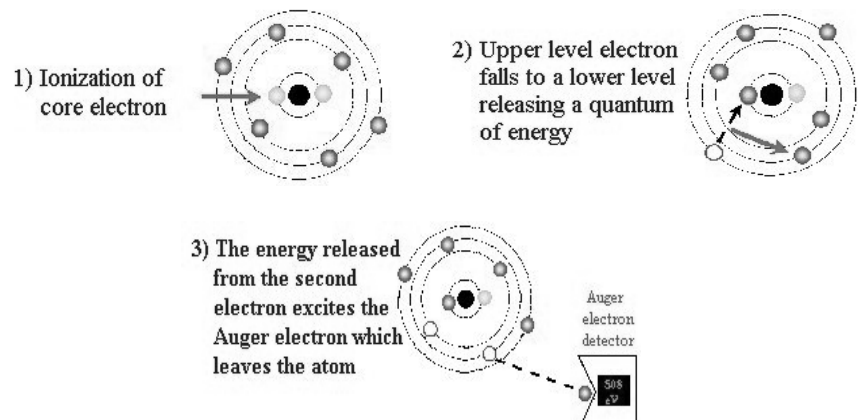

Figure1. Auger electron emission process

The kinetic energy of the Auger electron corresponds to the difference between the energy of the initial electronic transition and the ionization energy for the electron shell from which the Auger electron was ejected. These energy levels depend on the type of atom and the chemical environment in which the atom was located. Auger electron spectroscopy involves the emission of Auger electrons by bombarding a sample with energetic electrons and measures the intensity of Auger electrons as a function of the Auger electron energy. The resulting spectra can be used to determine the identity of the emitting atoms and some information about their environment. Since 1953, AES has become a practical and straightforward characterization technique for probing chemical and compositional surface environments.

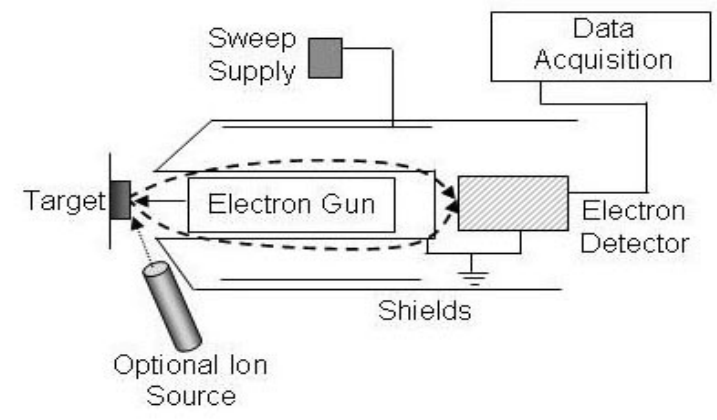

Figure 2.AES experimental set up

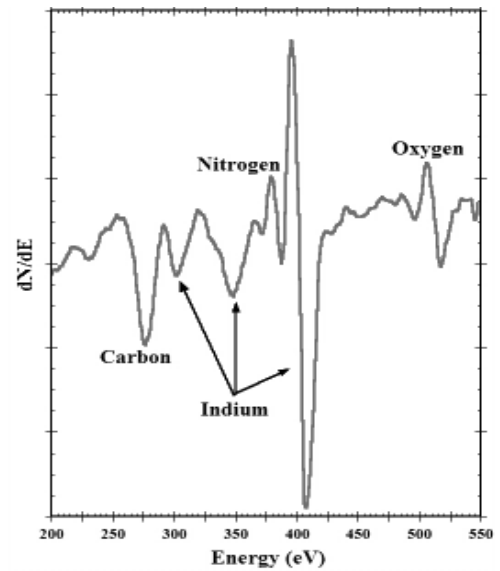

Figure 3. AES spectrum

AES is a popular technique for determining the composition of the top few layers of a surface. It cannot detect hydrogen or helium, but is sensitive to all other elements, being most sensitive to the low atomic number elements. 


\section{Low Energy Electron Diffraction (LEED)}

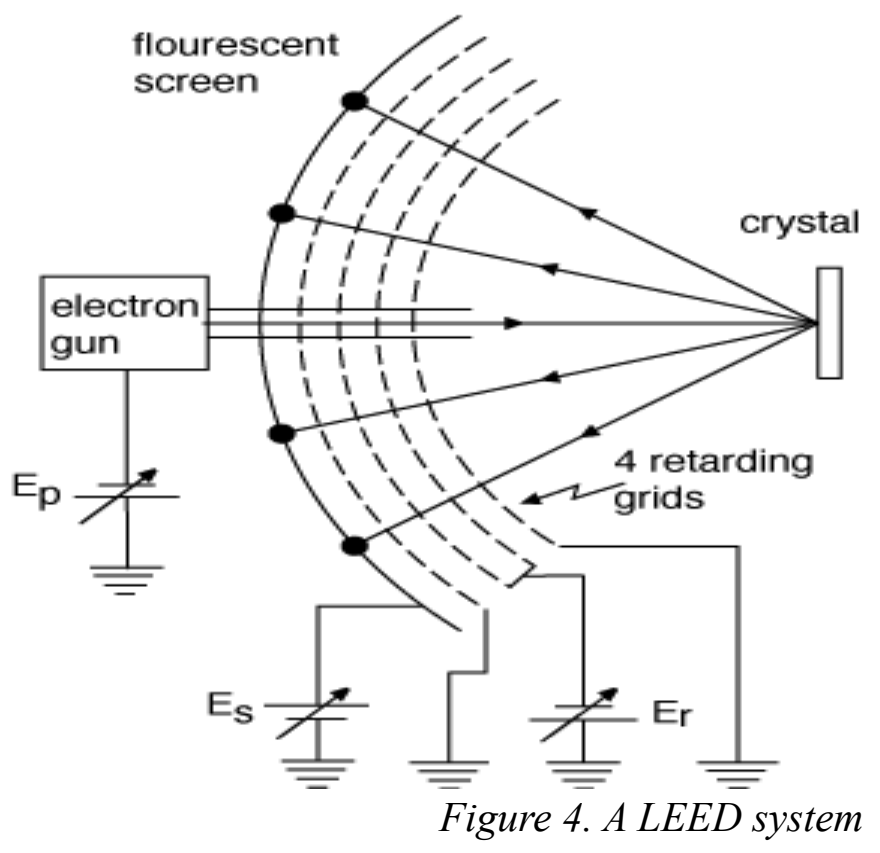

In this technique, a collimated beam of electrons is incident on the surface of the crystalline material. The spots of the diffracted electrons are observed on the fluorescent screen and the surface structure of the material is determined. The electron gun emits the electrons and accelerates them making a fine beam towards the sample. The detector consists of four metal grids at different voltages and a fluorescent screen. The first grid (counted from the sample) is on ground potential to ensure a field free region around the sample. The next two grids are set to the so-called retarding voltage. This voltage is slightly lower than the kinetic energy of the electrons produced by the gun. It repels almost all the inelastically scattered electrons. The elastically scattered electrons pass the next grid which is set to ground voltage again and are then accelerated towards the fluorescent screen which is set to a high positive voltage. Behind the screen there is a window in the vacuum system so that the LEED pattern can be observed directly or recorded with a video camera.

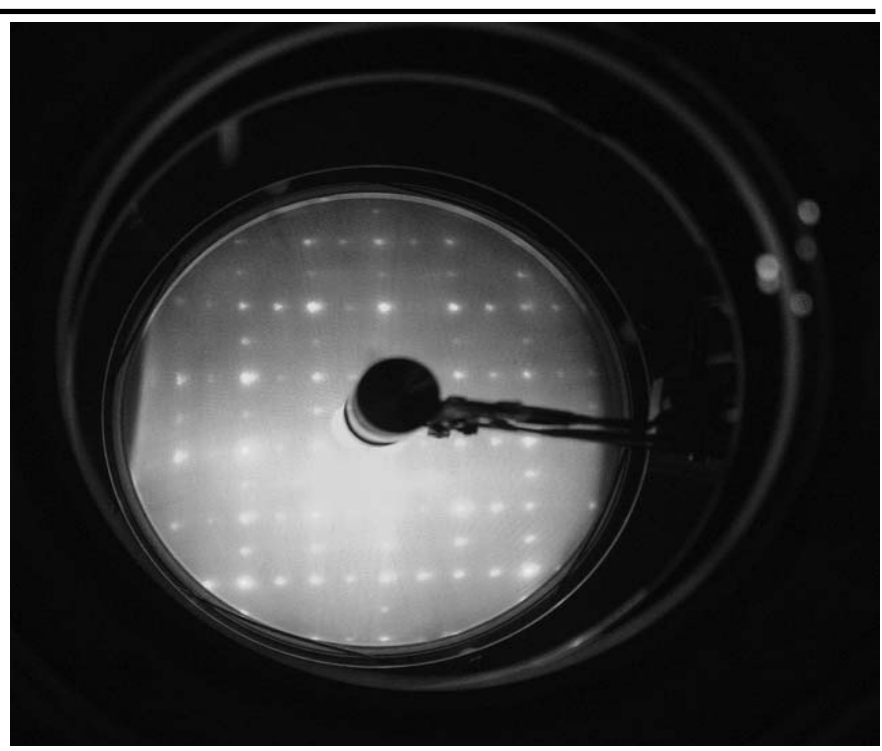

Figure 5. A LEED pattern

\section{High Resolution Electron Energy Loss Spectroscopy (HREELS)}

HREELS is a vibrational spectroscopic technique in which a low energy electron is incident on the surface and inelastically scattered electrons are collected in a specular direction and analyzed. The loss energy of electron is less then $500 \mathrm{meV}$. The inelastic scattering of electrons from surfaces is utilized to study electronic excitations or vibrational modes of the surface or of molecules adsorbed to a surface

The electrons are generated in an electron source, by heating a tungsten cathode, which is encapsulated by a negatively charged so called repeller that prevents stray electrons from coming into the detector unit. The electrons can leave the source only through a lens system. The purpose of this system is to focus the electrons on the entrance of the monochromator unit, to get a high initial electron flux. The monochromator is usually a Concentric Hemispherical Analyzer. In more sensitive setups an additional pre-monochromator is used. The task of the monochromator is to reduce the energy of the passing electrons to some $\mathrm{eV}$ due to the help of electron lenses. It further lets only those electrons pass which have the chosen initial energy. The electrons are then focused by a lens 
The Himalayan Physics, Vol.1, No.1, May 2010

system onto the sample. The inelastically scattered electrons enter into the analyzer region through the lenses. The analyzer again allows only electrons of certain energies to pass to the analyzing unit. The spectrometer is in general a computer simulated design which tries to optimize the resolution while keeping an acceptable electron flux.

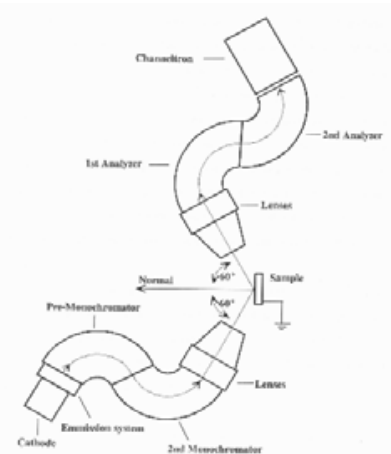

Figure 6. Schematic diagram sample

of the HREELS spectrometer

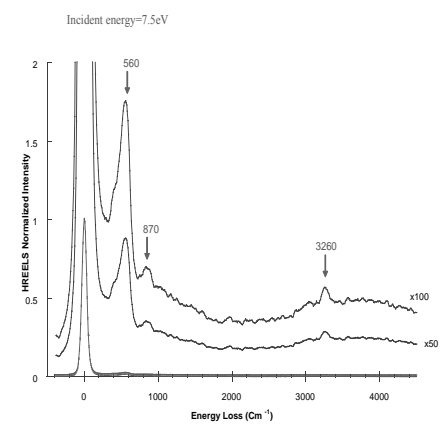

Figure 7. HREELS of InN
Figure 7 is the HREELS spectrum taken on a cleaned InN sample. We can see some of the loss features in the spectrum. From these loss features we can have information about the surface adsobates on the sample. A strong loss feature at $560 \mathrm{~cm}^{-1}$ is assigned to the Fuchs-Kliewer surface phonon. The loss features at $870 \mathrm{~cm}^{-1} \& 3260 \mathrm{~cm}^{-1}$ are assigned to $\mathrm{N}-\mathrm{H}$ bending and stretching modes of vibrations respectively. In a number of HREELS experiments, it has been reported that $\mathrm{InH}$ stretching vibration is seen at $1650-1700 \mathrm{~cm}^{-1}$ on InP surfaces. In this particular sample of InN, no loss features were observed in this range. It indicates that there is no surface InH and the film is N-Polar. So, HREELS is very important to know about the surface adsorbates and film polarity of the thin layers.

In conclusion, AES, LEED and HREELS are very helpful and important techniques in the area of surface science. From these techniques, we can study the composition, structure, vibrational modes of the surface adsorbates and the polarity of the thin films. 